\title{
Unity efficiency and zero-oscillations based MPPT for photovoltaic systems
}

\author{
Yacine TRIKI ${ }^{1,2,3}$, Ali BECHOUCHE ${ }^{1}$, Hamid SEDDIKI ${ }^{1}$ and Djaffar OULD ABDESLAM ${ }^{2 *}$ \\ PhD student, Dr., Prof., Dr. \\ ${ }^{1}$ L2CSP Laboratory, Mouloud Mammeri University of Tizi-Ouzou, Algeria \\ ${ }^{2}$ IRIMAS Institute, University of Haute Alsace, France \\ ${ }^{3}$ LAMPA Laboratory, Mouloud Mammeri University of Tizi-Ouzou, Algeria \\ *E-mail: djaffar.ould-abdeslam@uha.fr
}

* Corresponding author

\begin{abstract}
Maximum power point tracking (MPPT) is essential for photovoltaic systems to ensure a maximum power extraction from PV panels. Many MPPT techniques have been suggested in the literature. However, some issues such as oscillations, power loss and other technical aspects still unsolved. This paper presents and discusses a new MPPT algorithm with zero-oscillations and unity efficiency in transient and steady-states. This algorithm leads to track the maximum power point under extreme operating conditions. The proposed MPPT method is based on the simple adaptive linear neuron. In addition, its implementation is achieved without any additional control loop, which resulted in a simple control. In order to validate the proposal effectiveness, both simulation and experiment tests are carried out under variable irradiance and load. Comparison between the developed MPPT and the conventional perturb and observe algorithm is also performed. Obtained results show that with the proposed method, unity efficiency is reached and oscillations are fully removed in the transient and steady-states. The originality of this work is the design of a simple and efficient MPPT algorithm based on the ADALINE with unity efficiency and zero-oscillations. Moreover, the proposal is verified using a real PV system under irradiance and load changes.
\end{abstract}

Keywords: Adaptive linear neuron (ADALINE), DC-DC boost-converter, maximum power point tracking $(M P P T)$, perturb and observe ( $P \& O)$, photovoltaic system.

\section{INTRODUCTION}

Power generation from photovoltaic (PV) panels highly depends on weather conditions such as sunlight and temperature. So, a maximum power point tracking (MPPT) technique is needed to maximize the produced energy. Several algorithms have been suggested to track the maximum power point (MPP) with different levels in complexity, cost, convergence speed, steady-state oscillations, required sensors, and efficiency [1]. These algorithms can be sorted in conventional methods (CMs) and artificial intelligence (AI) based methods.

In $\mathrm{CMs}$, the most popular MPPT algorithms are the perturb and observe $(\mathrm{P} \& \mathrm{O})$ [2], [3], incremental conductance (IC) [4], [5], short circuit current [6] and open circuit voltage approaches [6]. Among these, $\mathrm{P} \& \mathrm{O}$ and IC are the extensively used methods due to their simplicity, acceptable performances and ease of implementation. These methods operate by increasing or decreasing the converter duty cycle in order to reach the MPP, which induce oscillations in the generated power. Moreover, they presented similar issues regarding losses in steady-state [7], and tracking performances under variable climatic conditions. As solution, adaptive CMs with variable step sizes have been developed [8]-[11]. In these strategies, when the operating point is far from the MPP, the step size is increased which enables to a fast-tracking ability. Otherwise, the step size is decreased gradually. Consequently, oscillations in the generated power become well reduced, which contribute to achieve a high efficiency [12]. These adaptive step size based MPPTs acquire a fast response and accurate steady-state performances. Nevertheless, some issues remain unsolved such as residual oscillations in steady-state and low tracking efficiency under variable environmental conditions. In addition, the heavy computational and strong non-linearity of the scaling factor restricts their application.

Recently, AI based MPPT algorithms are becoming the most used approaches as alternatives to the CMs due to their capability to resolving many problems such as convergence speed, oscillation around the MPP, and accuracy loss under fast change in atmospheric conditions. The well-known AI based MPPT algorithms are the approaches based on fuzzy logic (FL), neural networks (NNs), genetic 
algorithm (GA), and particle swarm optimization [1], [13]. These techniques lead to consistent MPPTs, which is due to their ability to manage nonlinear aspects of PV systems. The FL based MPPT has been largely investigated in PV systems control [14], [15]. This approach improves the dynamic tracking efficiency especially under variable weather conditions. However, its main disadvantage is that the operation point must be known precisely. Indeed, to achieve good performance, the input variables of membership functions, based on the PV system power curve, must be well tuned in different intervals [14]. This latter increases hardware and software complexity and leads to heavy implementation. Meanwhile, NN based MPPTs has become increasingly popular. This is due to the numerous advantages of NNs. Really, they can detect complex nonlinear relationships between input and output signals of the PV system; they don't require an accurate model; and they require less formal statistical training, simplicity and ease of implementation. Moreover, divers training algorithms is available which provide a large number of solutions [1]. Several NNs based MPPT algorithms have been proposed in the literature. For instance, a $\mathrm{P} \& \mathrm{O}$ algorithm assisted by an online learned $\mathrm{NN}$ is developed in [16] to solve its low performances under fast changing in solar irradiance. In [17], a variable step-size MPPT based on extension $\mathrm{NN}$ is suggested. A radial basis function network based MPPT is proposed in [18]. In [19], a combination of NN and FL with polar information controller is suggested. Gas are used for optimizing the offline trained NN in [20]. Although these NN based MPPT algorithms can provide better MPPT performances, some of them presented complex structures [17]-[20], and required repetitive offline training with solar irradiance and cells temperature measurement [1], [20]-[23]. This can mainly contribute to increase their implementation cost in digital signal processors.

Despite the adaptive CMs and AI based MPPT algorithms have been proposed for improvement, they presented some drawbacks such as oscillatory behavior around the MPP and important losses in steady-states. In this sense, some MPPT algorithms have been developed to improve steady-state behavior and provide an accurate tracking under variable environmental conditions [24]-[27]. In [24] and [25], modified IC and P\&O methods which reduced, respectively, steady-state oscillations and improved tracking efficiency, are suggested. In [26], the authors presented an improved MPPT algorithm with zero-oscillation. This MPPT is implemented in two stages, the first is based on adaptive scaling factor beta to improve transient response; and the second is based on a modified $\mathrm{P} \& \mathrm{O}$ to eliminate steady-state errors. In [27], three techniques are combined to improve steady-state behavior and transient operation. The aforementioned methods show good performances; however, some proposed algorithms presented complex structures [24], [26], [27]. In addition, their validations are performed using PV emulators, which remain non-real PV systems.

General requirements of an MPPT are the simplicity, low cost, low output power fluctuation, and quick tracking under variable operating conditions. To meet these requirements a novel adaptive linear neuron (ADALINE) based MPPT (AbM) is proposed in this paper. The main advantages of the ADALINE are its simple structure, convergence speed, filtering capability and ability to be trained online [28]. The proposed AbM strategy is implemented for a simplification purpose without any additional control loop. In order to corroborate the theoretical findings, simulation and experimental tests are conducted with different irradiance conditions (dynamic and level) and connected loads. A comparison with the $\mathrm{P} \& \mathrm{O}$ algorithm is performed under the same conditions. Indeed, the $\mathrm{P} \& \mathrm{O}$ algorithm will be adopted as a comparison method in this paper. This choice is motivated by the fact that the P\&O is the most popular technique applied to track the MPP in practice [3], [21], [25] [29], [30] and adopted by many authors for comparison [2], [3], [10], [21]. This algorithm is easy to implement and can be applied to all PV modules. It does not require previous knowledge of the PV system or environmental conditions measurement. Moreover, it exhibits satisfactory performances with fast convergence towards the MPP. The obtained results from the comparative study with the $\mathrm{P} \& \mathrm{O}$ show perfect performances of the proposed method in transient and steady-states. The originality of this work is the built of an efficient and simple MPPT algorithm based on the ADALINE which acts as an adaptive filter. So, the proposal leads to achieve an MPPT algorithm with unity efficiency and zero-oscillations, and can works even under sudden changes in solar irradiance. Moreover, unlike many others methods, the proposed method is verified using a real PV system under variable irradiance and load.

This paper is organized as follows. The general structure of the controlled PV system is described in the second section. In the third section, the proposed control strategy for MPPT is 
provided. The forth section presented and discussed the simulation results. Comparison results with other advanced zero-oscillations based MPPT algorithms are reported in the fifth section. Obtained experimental results are illustrated and discussed in the sixth section. Finally, the seventh section concludes this paper.

\section{PV SYSTEM DESCRIPTION}

The general structure of the PV system is presented in Fig. 1. It consists on a PV panel, boost converter, control unit and applied load. The measured PV panel voltage and current are fed into the MPPT algorithm, and subsequently, the generated output duty cycle is used to drive the power switch. It can be seen that there is no additional control loop in the system which enables to reduce the complexity.

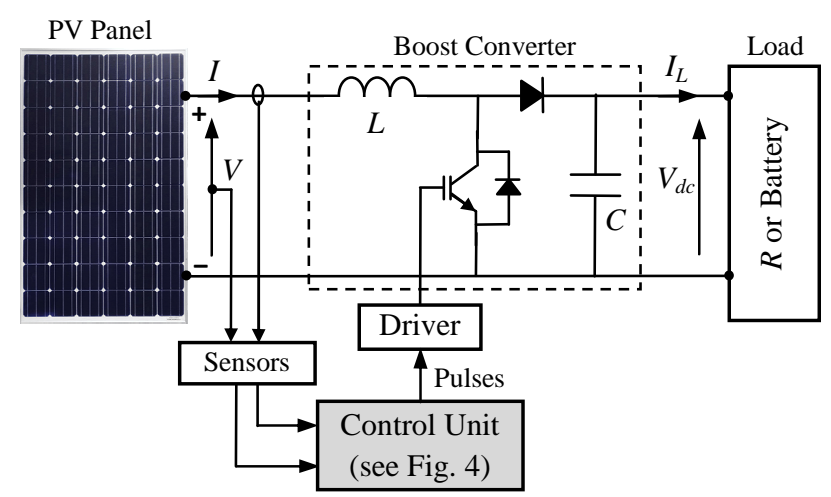

Fig. 1. Architecture of the controlled PV system.

The converter output can be connected to a battery load, to a regulated DC-bus voltage or to a simple scenario of DC resistive.

\section{1) PV panel}

For analysis, the adopted PV panel equivalent circuit is the single diode model. This circuit consists on a current source with generated photocurrent $I_{p h}$ directly proportional to the sun irradiance, a diode, a series resistance $R_{s}$ and a shunt resistance $R_{p}$. The I-V relationship of a PV panel is given by the following expression:

$$
I=I_{p h}-I_{o}\left[\exp \left(\frac{q\left(V+R_{s} I\right)}{a k T N_{s}}\right)-1\right]-\frac{V+R_{s} I}{R_{p}}
$$

where $I_{o}$ is the diode reverse saturation current, $a$ is the diode ideality factor, $q$ is the electron charge, $k$ is the Boltzmann constant, $N_{s}$ is the number of cells connected in series and $T$ is the cell's temperature in Kelvin. The photocurrent $I_{p h}$ mainly depends on the solar irradiance. Its expression can be written as

$$
I_{p h}=\frac{\left(I_{s c}+K_{i} \Delta_{T}\right) G}{G_{n}}
$$

where $I_{s c}$ is the PV panel short-circuit current at nominal temperature and solar irradiance, $G$ is the solar irradiance on the device surface, $G_{n}$ is the nominal solar irradiance, $K_{i}$ is the temperature coefficient and $\Delta_{T}=T-T_{n}$ where $T$ and $T_{n}$ are, respectively, the measured and nominal cell's temperatures.

According to the adopted PV model, the current-voltage and power-voltage characteristics of the used IFRI260-60 PV panel are shown in Fig. 2. 

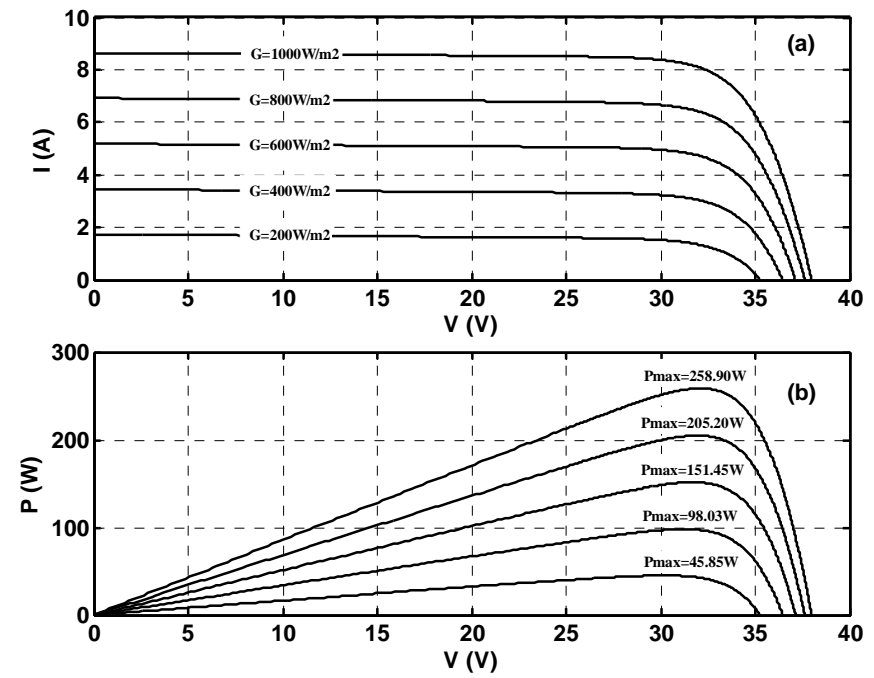

Fig. 2. Characteristics of the used IFRI $260-60 \mathrm{PV}$ panel at $25^{\circ} \mathrm{C}$ for different solar irradiance levels (graphs plotted with step of $200 \mathrm{~W} / \mathrm{m}^{2}$ ): (a) current-versus-voltage; (b) power-versus-voltage.

\section{2) DC-DC boost converter}

In PV power generation systems, DC-DC boost converters are widely used as an adaptation stage between the PV panel and the load. Based on the information supplied by the MPPT algorithm, an optimal duty-cycle is generated to maximize the solar power available on the PV panel. The control is normally achieved by pulse-width modulation (PWM) technique with a fixed switching frequency. Topology of the used DC-DC Boost converter is schematized in Fig. 1.

At high switching frequency, average model of the DC-DC boost converter can be derived. In resistive load or DC-bus voltage configurations, the dynamic model of the converter is described by the following differential equations:

$$
\begin{aligned}
& \frac{d I}{d t}=\frac{V}{L}-\frac{V_{d c}}{L}(1-\alpha) \\
& \frac{d V_{d c}}{d t}=\frac{I}{C}(1-\alpha)-I_{L}
\end{aligned}
$$

where $V$ is the input voltage, $I$ is the input current, $V_{d c}$ is the output voltage, $\alpha$ is the duty cycle, $I_{L}$ is the load current, and $C$ is the DC-bus capacitance.

In case of battery load, the boost converter is described by the following equations:

$$
\begin{aligned}
\frac{d I}{d t} & =\frac{V}{L}-\frac{V_{b}}{L}(1-\alpha) \\
I_{b} & =I(1-\alpha)-I_{L}
\end{aligned}
$$

where $V_{b}$ and $I_{b}$ denote the battery voltage and current, respectively.

\section{CONTROL STRATEGY}

\section{1) ADALINE theory}

The ADALINE was first studied by Windrow and his colleagues in the 1960s [28]. Due to its simple structure and ability to be trained online, ADALINE has become a powerful tool used in many applications such as identification [31] and control [32]. ADALINE is a multi-input - single-output structure which is equivalent to one neuron. Fig. 3 shows the ADALINE architecture. It's composed by an input vector $X(k)=\left[x_{I}(k) \ldots x_{m}(k)\right]$ of $m$ dimension, an adjustable weight vector $W(k)=\left[w_{1}(k)\right.$ $\left.\ldots w_{m}(k)\right]^{T}$ of $m$ dimension, a linear activation function and a single estimated output $y_{e s t}(k)$. 


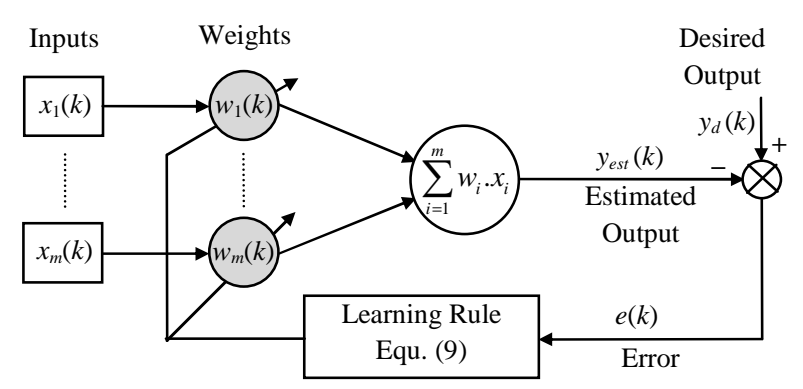

Fig. 3. Architecture of the ADALINE. as follows:

The estimated output of the ADALINE can be calculated for any input $X(k)$ at sample time $k$

$$
y_{\text {est }}(k)=\sum_{i=1}^{m} w_{i}(k) \cdot x_{i}(k)
$$

ADALINE is an online learning process. Its weights are adjusted to minimize the error $e(k)$ between the estimated output $y_{\text {est }}(k)$ and the desired response $y_{d}(k)$. The estimation error $e(k)$ is then defined as:

$$
e(k)=y_{d}(k)-y_{e s t}(k)=y_{d}(k)-\sum_{i=1}^{m} w_{i}(k) \cdot x_{i}(k) .
$$

Learning process means the procedure of weights updating. When inputs are applied to the network, its output $y_{e s t}(k)$ is compared to a target $y_{d}(k)$. Based on the generated error, a learning rule is used to adjust the weights in order to move the ADALINE output closer to the target. The most known learning rule is that called $\alpha$-LMS algorithm given as follows:

$$
W(k+1)=W(k)+2 \mu e(k) X(k)
$$

where $\mu$ is the learning rate. Its value affects directly the accuracy, convergence speed and stability of the ADALINE. Indeed, low value of $\mu$ leads to increase the accuracy and the stability at cost of slower convergence speed. On the other hand, high value of $\mu$ leads to high convergence speed but with less accuracy and stability. So, the choice of $\mu$ is a tradeoff between the stability and convergence speed of the ADALINE [28]. For input pattern vectors, stability is ensured for most practical purposes if

$$
0<\mu<2 \text {. }
$$

\section{2) Proposed ADALINE based MPPT (AbM)}

As shown in Fig. 2(b), for each operating condition, there is a unique MPP located at the knee of the $I(V)$ curve [see Fig. 2(a)]. In other words, the MPP is reached when the derivative of $P$ according to $V$ is null. Therefore, the following equation must be verified:

$$
V=-I \frac{d V}{d I} \text {. }
$$

In the other side, from (3), the input voltage of the boost converter is given by:

$$
V=L \frac{d I}{d t}+V_{d c}(1-\alpha)
$$

The MPP is reached when the input voltages of the DC-DC boost converter meet that given by equation (11). Hence:

$$
L \frac{d I}{d t}+V_{d c}(1-\alpha)=-I \frac{d V}{d I} .
$$

The quantity $L d I / d t$ can be neglected (it worth zero in steady-state). In transient-state, the sun irradiance variation is often slow so, the current derivative takes low value. Moreover, the proposed MPPT algorithm is faster than the current variation. Using this assumption and equation (13), the optimal duty cycle at the MPP is then given by:

$$
\alpha=\frac{I d V}{V_{d c} d I}+1 .
$$


The discrete form of the above equation can be expressed as follows:

$$
\alpha(k)=\frac{I(k) \Delta V(k)}{V_{d c}(k) \Delta I(k)}+1 .
$$

At this stage, the ADALINE concept is introduced to solve equation (15) in the aim to find the optimal duty cycle. Then, the following equalities are posed:

$$
\begin{gathered}
\frac{I(k) \Delta V(k)}{V_{d c}(k) \Delta I(k)}+1=y_{d}(k) \\
\alpha(k)=w(k)=y_{\text {est }}(k) \\
x(k)=1 .
\end{gathered}
$$

Based on (16)-(18) and (9), the adaptive MPPT algorithm is derived as follows:

$$
\begin{aligned}
\alpha(k+1) & =\alpha(k)+2 \mu e(k) \\
& =\alpha(k)+2 \mu\left[\frac{I(k) \Delta V(k)}{V_{d c}(k) \Delta I(k)}+1-\alpha(k)\right] .
\end{aligned}
$$

After the learning process, the error $e(k)$ becomes low and the optimal duty cycle is then obtained. According to (19), the diagram of the proposed AbM strategy is shown in Fig. 4.

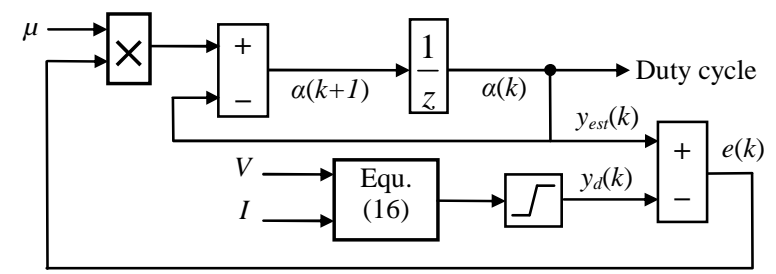

Fig. 4. Block diagram of the proposed AbM algorithm.

As seen in (20), to evaluate the desired output $y_{d}(k)$, the $V_{d c}(k)$ knowledge is required. The last can be estimated in steady-state as follows:

$$
V_{d c}(k)=\frac{V(k)}{1-\alpha(k)} .
$$

Consequently, there is no additional sensor for the proposed method.

\section{SIMULATION RESULTS}

To verify performances of the proposed AbM, the overall system is set for digital simulation in MATLAB/Simulink environment. The results are compared to those obtained by the conventional $\mathrm{P} \& \mathrm{O}$ algorithm. In a first scenario, dynamic and steady-state performances are examined according to fast $\left(200 \mathrm{~W} / \mathrm{m}^{2} / \mathrm{s}\right)$ and slow $\left(20 \mathrm{~W} / \mathrm{m}^{2} / \mathrm{s}\right)$ solar irradiance slop change. The solar irradiance $G$ changes between 200 and $1000 \mathrm{~W} / \mathrm{m}^{2}$. The output voltage of the boost converter is fixed to $V_{d c}=300 \mathrm{~V}$. This situation occurs when the converter operates as a battery charger or supplies a DC bus voltage. In a second scenario, the proposed algorithm is tested under fixed solar irradiance $\left(1000 \mathrm{~W} / \mathrm{m}^{2}\right)$, meanwhile, the DC bus voltage is varied between 250 and $350 \mathrm{~V}$. This situation occurs, for instance, when the converter supplies a grid connected inverter. In this case, the inverter introduces a disturbance on the DC bus voltage. The two tests sequences are illustrated in Figs. 5(a) and (b). 

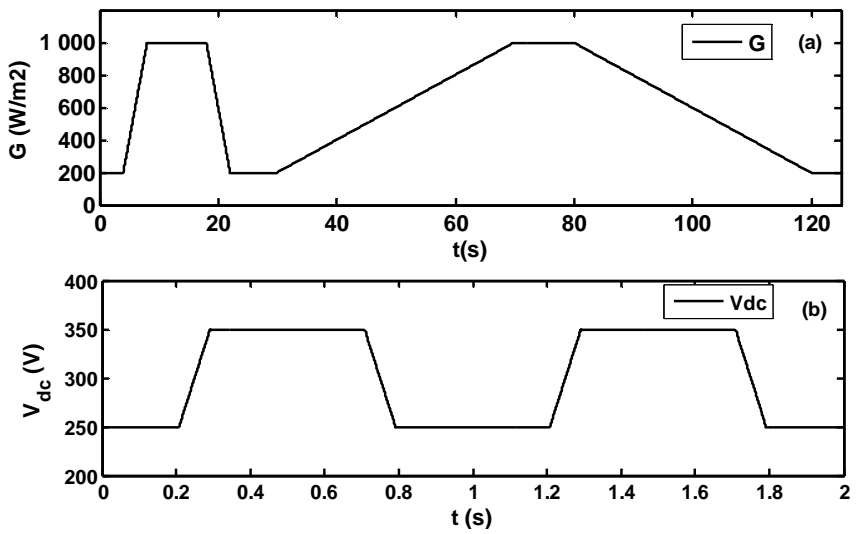

Fig. 5. Simulation test sequences: (a) Fast and slow solar irradiance profile with a fixed DC bus voltage $\left(V_{d c}=300 \mathrm{~V}\right)$; (b) variable DC bus voltage and fixed solar irradiance $G=1000 \mathrm{~W} / \mathrm{m}^{2}$.

The AbM learning rate $\mu$ and the $\mathrm{P} \& \mathrm{O}$ algorithm step size are optimized through simulation tests according to steady state accuracy and tracking speed requirement. From (10), an optimal choice of the AbM learning rate $\mu$ which the same as the ADALINE learning rate in (9) can be achieved according to the following considerations. High value of $\mu$ leads to high convergence speed but with less stability and accuracy. This mainly causes oscillatory behavior during the MPP tracking. So, correct tracking of the MPP can be lost. On the other way, low value of $\mu$ leads to more stability and accuracy at cost of slow tracking speed. Accordingly, correct choice of $\mu$ is a tradeoff between the oscillatory behavior, stability and the tracking speed of the proposed MPPT. In this work and according to these considerations, $\mu$ is experimentally adjusted to 0.015 through a trial-and-error approach. In the same way, a correct choice of the P\&O step size $\Delta \alpha$ is a tradeoff between the transient and steady states performances. Indeed, high step size contributes to a faster response while more power losses are caused in steady state. Opposite situation is obtained when a low $\Delta \alpha$ is chosen. In this work, the $\mathrm{P} \& \mathrm{O}$ algorithm step size is experimentally adjusted to 0.0025 through a trial-anderror approach. The overall system parameters including the used IFRI260-60 PV panel at standard test conditions (STC) are given in Table 1.

Table 1

PV system parameters

\begin{tabular}{|c|c|c|}
\hline \multicolumn{3}{|c|}{ PV panel IFRI260-60 at STC } \\
\hline$P_{\max }$ & Maximum power & $260 \mathrm{~W}$ \\
\hline$I_{s c}$ & Short-circuit current & $8.65 \mathrm{~A}$ \\
\hline$V_{o c}$ & Open-circuit voltage & $38.1 \mathrm{~V}$ \\
\hline$N_{s}$ & Number of cells in series & 60 \\
\hline \multicolumn{3}{|c|}{ Boost converter } \\
\hline$f_{s}$ & Switching frequency & $100 \mathrm{KHz}$ \\
\hline$L$ & Inductance & $5 \mathrm{mH}$ \\
\hline$C$ & Capacitor & $3300 \mu \mathrm{F}$ \\
\hline \multicolumn{3}{|c|}{ MPPT algorithm } \\
\hline$\mu$ & AbM learning rate & 0.015 \\
\hline$\Delta \alpha$ & P\&O step size & 0.0025 \\
\hline$T_{e}$ & Sampling period & $0,0001 \mathrm{~s}$ \\
\hline \multicolumn{3}{|c}{} \\
\hline
\end{tabular}

\section{1) Performances under variable solar irradiance $\left(V_{d c}=300 \mathrm{~V}\right)$}

The AbM algorithm is tested with a solar irradiance change according to the test sequences given in Fig. 5(a). The generated powers from the PV panel are plotted in Fig. 6. From Fig. 6(a), it seems that the MPP is correctly tracked using both methods. However, a zoom in steady-state, given in Fig. 6(b), indicates that the AbM algorithm is clearly better than the P\&O algorithm. Indeed, there are no oscillations in the power with the proposed method. Moreover, it achieves $100 \%$ of efficiency. The generated power in steady-state is $258.90 \mathrm{~W}$. This value corresponds to the theoretical optimum of 
258.90W. A zoom in transient-state of the produced power is given in Fig. 6(c). Superiority tracking of the AbM algorithm is also observed during the transient-state (or during the solar irradiance change) where $100 \%$ of efficiency is reached with the proposal. However, the $\mathrm{P} \& \mathrm{O}$ algorithm exhibits oscillations and power loss.
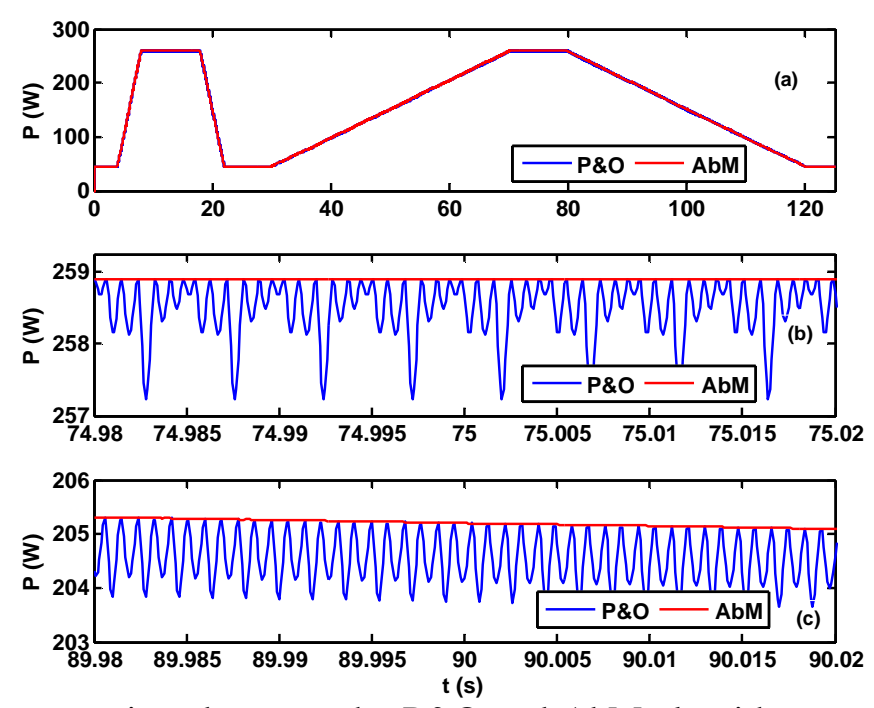

Fig. 6. Performances comparison between the $\mathrm{P} \& \mathrm{O}$ and $\mathrm{AbM}$ algorithms under fast and slow solar irradiance profile: (a) output power of the PV panel; (b) zoom in the output power of the PV panel in steady-state; (c) zoom in the output power of the PV panel in transient-state.
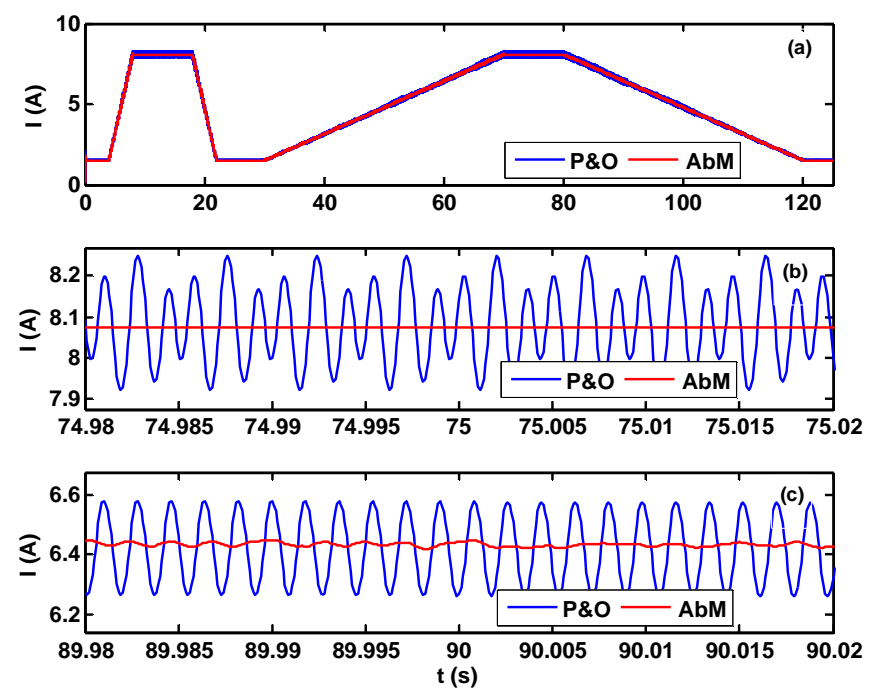

Fig. 7. Performances comparison between the $\mathrm{P} \& \mathrm{O}$ and $\mathrm{AbM}$ algorithms under fast and slow irradiance profile: (a) PV output current; (b) zoom in the PV output current in steady-state; (c) zoom in the PV output current in transient-state.

Fig. 7 shows the PV output current under the different atmospheric conditions. From Fig.7 (a), it is appearing that the optimal current has a rapid response and track correctly the solar irradiance. The results given in Fig. 7(b) clearly show that the suggested AbM algorithm has a better performance in steady-state compared to the conventional P\&O. The current oscillation rates $(\Delta I / I)$ for the $\mathrm{P} \& \mathrm{O}$ and the $\mathrm{AbM}$ is, respectively, $4 \%$ and $0 \%$. Hence, the proposal fully removes the oscillations in the current which leads to thermal stress elimination in the PV panel [33]. During the solar irradiance change, the current oscillations for the $\mathrm{P} \& \mathrm{O}$ and $\mathrm{AbM}$ is, respectively, $4 \%$ and $<0.5 \%$.

Fig. 8 shows the output voltage of the PV panel under variable solar irradiance conditions. As shown in Fig 8(a), in term of oscillations, the AbM is much better than the P\&O. Indeed, a zoom in steady-state (see Fig 8(b)) reveals a complete absence of oscillations in the voltage $(\Delta V / V=0 \%)$ using the AbM. Meanwhile, in case of the $\mathrm{P} \& \mathrm{O}$, the obtained voltage oscillation is $2.3 \%$. Therefore, the 
issues regarding losses and oscillations in steady-state are definitively solved by the proposal. During the solar irradiance change, the voltage oscillations for the $\mathrm{P} \& \mathrm{O}$ and the $\mathrm{AbM}$ is, respectively, $2.4 \%$ and $<0.5 \%$ (Fig. 8(c)).
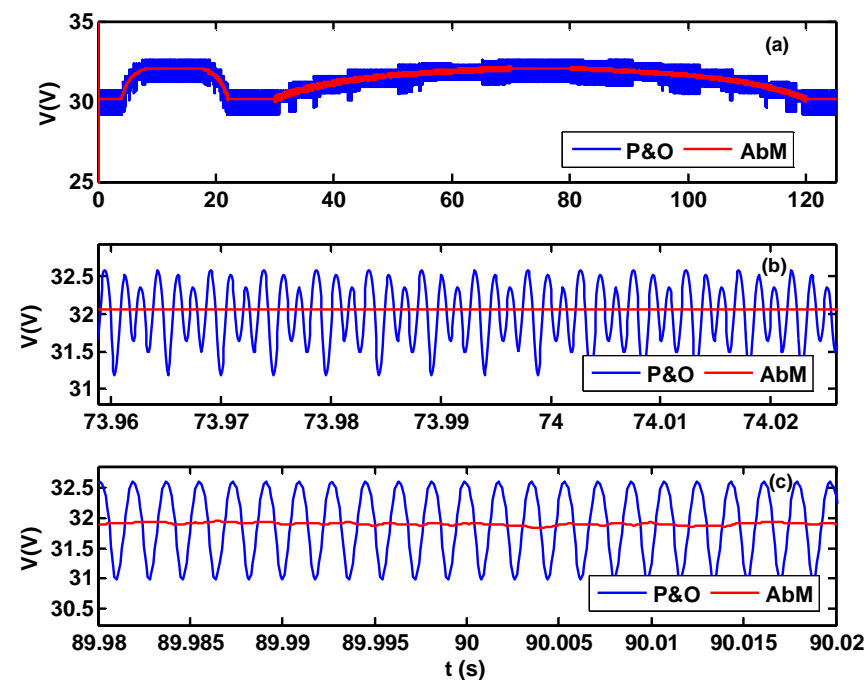

Fig. 8. Performances comparison between the $\mathrm{P} \& \mathrm{O}$ and $\mathrm{AbM}$ algorithms under fast and slow solar irradiance profile: (a) PV output voltage; (b) zoom in the PV output voltage in steady-state; (c) zoom in the PV output voltage in transient-state.
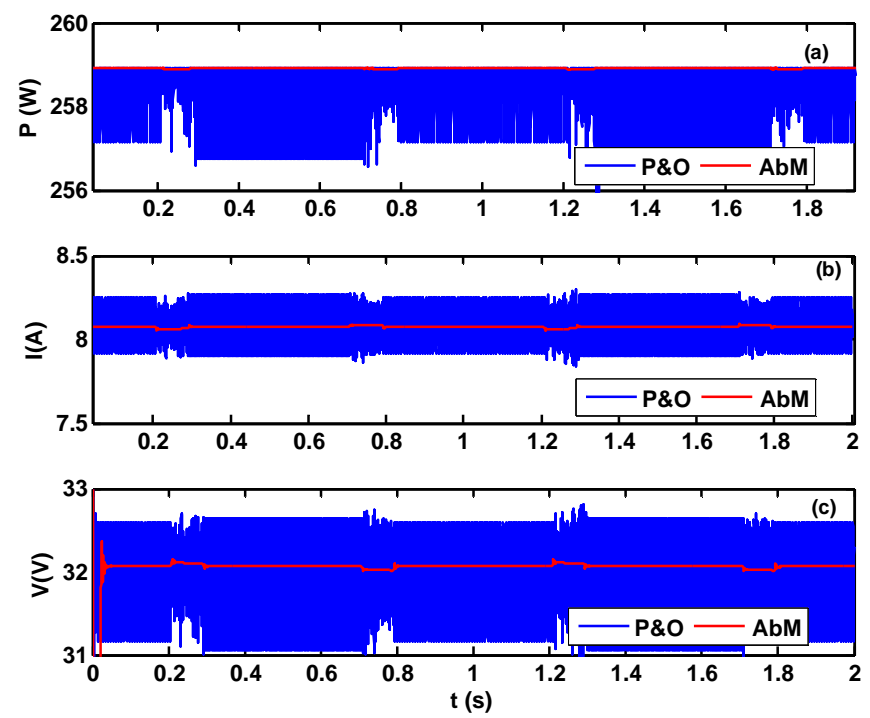

Fig. 9. Performances comparison between the $\mathrm{P} \& \mathrm{O}$ and $\mathrm{AbM}$ algorithms under variable $\mathrm{DC}$ bus voltage: (a) PV output power; (b) PV output current; (c) PV output voltage.

\section{2) Performances under variable DC bus voltage $\left(G=1000 \mathrm{~W} / \mathrm{m}^{2}\right)$}

Fig. 9 exhibits performances comparison between the two algorithms according to the test sequences given in Fig. 5(b). When variation occurs in the DC bus voltage, the AbM algorithm has a stable output signals compared to the output signal obtained by the $\mathrm{P} \& \mathrm{O}$.

As shown in Fig. 9(a), the generated power using the proposed control still 100\% despite the DC bus voltage variation. Figs. 9(b) and (c) display the current and voltage of the PV panel, respectively. It can be seen that there are no oscillations in the both quantities in steady-state with the suggested algorithm. Practically, in term of oscillations, similar results are reached during $V_{d c}$ change.

\section{COMPARISON STUDY}

So far, performances comparison of the proposed AbM is performed against the conventional $\mathrm{P} \& \mathrm{O}$ algorithm. This provides a reference point to compare with advanced MPPT algorithms. In this 
sense, comparative study between the proposal and other new advanced zero-oscillations based MPPT algorithms [24]- [27] is reported in Table 2. The selected algorithms are presented for their advanced features that allow a fast-tracking efficiency with zero-oscillations. In [24], the authors propose an IC method based on the switching ripple detection. The proposal uses a digital lock-in amplifier to extract amplitude of the oscillation ripple even under a noise presence. In [25], a modified P\&O based on an adaptive perturbation size with a dynamic boundary condition is proposed. This proposal minimizes the loss due to the direction losing. In [26], an improved MPPT algorithm with zero oscillation is presented. Its implementation is performed through two stages. The first stage is based on adaptive scaling factor beta to improve transient response and the second one is based on a modified $\mathrm{P} \& \mathrm{O}$ to eliminate steady-state errors. In [27], three techniques are combined to improve steady-state behavior and transient operation; the first one is the idle operation on the MPP that is used to steady-state oscillation cancellation; the second one is an identification of the solar irradiance change through a natural perturbation to eliminate the confusion caused by the solar irradiance change; and the third is a simple multi-level adaptive tracking step that is used to obtain an accurate tracking.

The aforementioned methods show good performances; however, some proposed algorithms presented a complex structure [24]- [26]. Moreover, their validation is performed using PV emulators [24]- [27], which remain non-real PV systems. As it can be seen in Table 2, superiority of the proposed AbM is clearly established in terms of efficiency oscillations and simplicity compared than the advanced zero-oscillations based MPPT algorithms.

Table 2

Comparison between the P\&O, proposed methods in [24]-[27], and the AbM

\begin{tabular}{|c|c|c|c|c|}
\hline & \multicolumn{2}{|c|}{ Efficiency } & \multirow[b]{2}{*}{$\begin{array}{l}\text { Tracking } \\
\text { Speed }\end{array}$} & \multirow[b]{2}{*}{ Notes } \\
\hline & $\begin{array}{l}\text { Steady } \\
\text { state }\end{array}$ & $\begin{array}{l}\text { Transient } \\
\text { state }\end{array}$ & & \\
\hline $\mathrm{P} \& \mathrm{O}$ & $99 \%$ & $99 \%$ & Slow & - Baseline comparison algorithm \\
\hline$[24]$ & $99 \%$ & $98 \%$ & Fast & $\begin{array}{l}\text { - IC method based on the switching ripple detection; } \\
\text { - The validation is made with a PV emulator. }\end{array}$ \\
\hline$[25]$ & $99.4 \%$ & $98.2 \%$ & Fast & $\begin{array}{l}\text { - Modified P\&O based on an adaptive perturbation size; } \\
\text { - The validation is made with a PV emulator. }\end{array}$ \\
\hline$[26]$ & $99.98 \%$ & $99.83 \%$ & Medium & $\begin{array}{l}\text { - Complex structure (combination of two methods); } \\
\text { - The validation is made with a PV emulator }\end{array}$ \\
\hline$[27]$ & $\approx 100 \%$ & $99.3 \%$ & Fast & $\begin{array}{l}\text { - Complex structure (combination of three techniques); } \\
\text { - The validation is made with a PV emulator }\end{array}$ \\
\hline $\mathrm{AbM}$ & $100 \%$ & $100 \%$ & Very Fast & $\begin{array}{l}\text { - Based on the simple ADALINE; } \\
\text { - The validation is made using a real PV system. }\end{array}$ \\
\hline
\end{tabular}

\section{EXPERIMENTAL RESULTS}

The proposed AbM algorithm has been also evaluated by experiment. The test bench is depicted in Fig. 10, which is made up of an IFRI260-60 PV panel, boost converter, load resistance and measurement tools. The developed $\mathrm{AbM}$ as well as the $\mathrm{P} \& \mathrm{O}$ algorithms are implemented under MATLAB/Simulink environment and executed on a dSPACE DS1104 board.

On the PV panel side, artificial solar irradiance is provided by four halogen lamps. Their luminous intensity is controlled using a single-phase power controller. A light radiation detector is used for irradiance measurement at the PV panel surface. Using a same setting as the simulations, the dynamic test sequences is composed by consecutive fast and slow increased and decreased solar irradiance ramps. Due to the low halogen lamps capability, the maximum irradiance level obtained at the PV panel surface is around $500 \mathrm{~W} / \mathrm{m} 2$. The boost converter supplies a load resistance $(R=330 \Omega)$. Consequently, a simultaneous change of $G$ and $V_{d c}$ is achieved during the experiment. The waveforms of the test sequences are plotted in Fig. 11(a) and (b). 

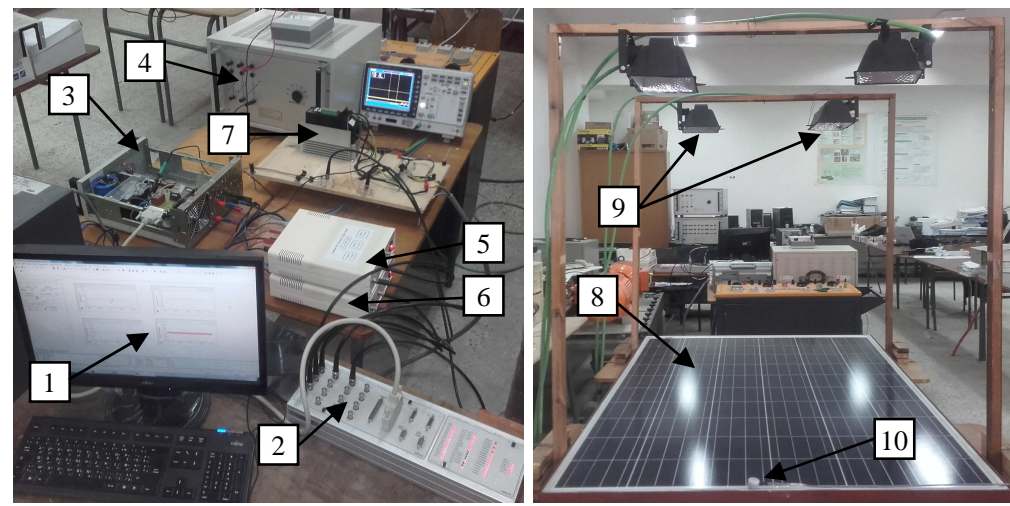

Fig. 10. View of the experimental test bench: (1) PC-Pentium + dSPACE board+ ControlDesk; (2) dSPACE input/output connectors; (3) DC-DC boost converter; (4) load resistance; (5) voltage sensor; (6) current sensor; (7) single phase power controller; (8) PV panel; (9) halogen lamp; and (10) irradiance sensor.
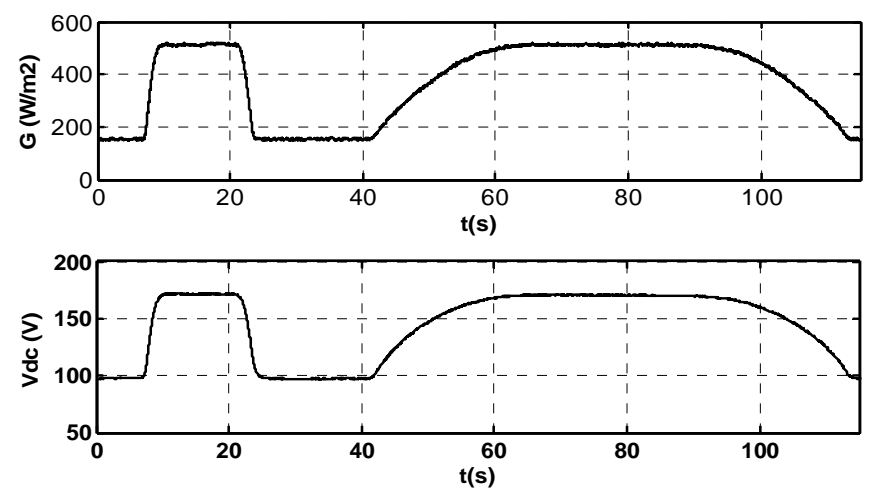

Fig. 11. Experimental test sequences: (a) irradiance profile reproduced in the laboratory by using halogen lumps; (b) Variation of $V_{d c}$ induced by the irradiance change.
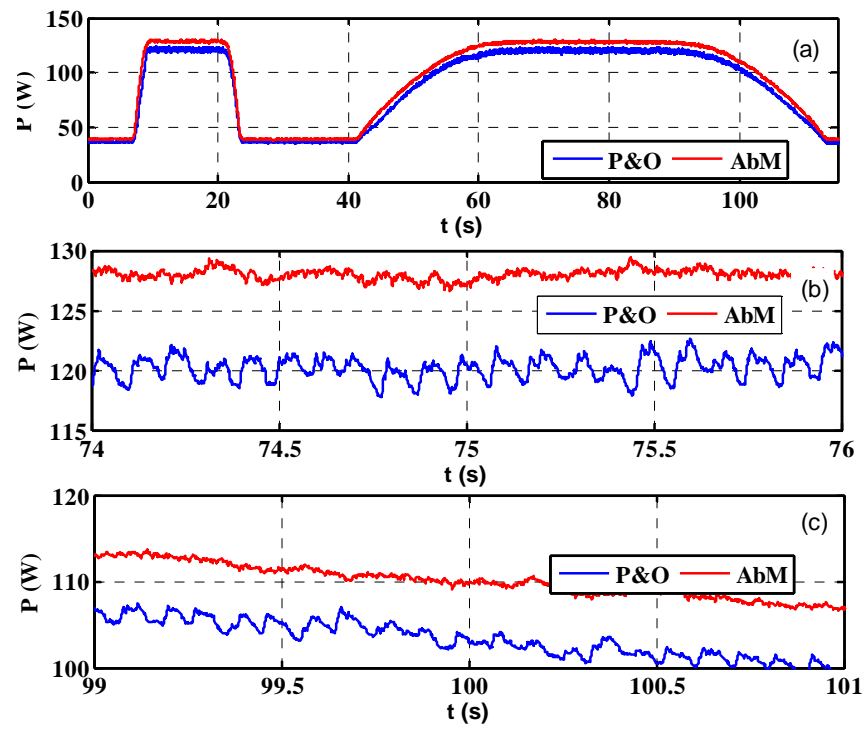

Fig. 12. Experimental comparison results of the $\mathrm{AbM}$ and $\mathrm{P} \& \mathrm{O}$ methods: (a) PV power tracking performances; (b) zoom on the PV power at steady state; (c) zoom on the PV power in transient state.

Fig. 12 gives output power of the PV panel when the irradiance profile, shown in Fig. 11(a), is applied. From Fig. 12(a), it can be seen that the new AbM algorithm is able to track correctly the irradiance change. Moreover, it shows better performances compared to the conventional P\&O algorithm. At high irradiance level, the extracted power in steady-state by the AbM and P\&O are, respectively, 128W and 120W (see Fig. 12(b)). Hence, AbM method increases the output power by 
$6.67 \%$. At low irradiance level, the output powers in steady-state are, respectively, $39 \mathrm{~W}$ and $36 \mathrm{~W}$. In this case, the proposed method increases the power by $8.33 \%$. In transient-states (during fast and slow irradiance change), superiority of the proposed method is clearly displayed in Fig. 12(c). Moreover, the obtained results clearly demonstrates that the AbM has a better signal waveform and reduced oscillations compared to the $\mathrm{P} \& \mathrm{O}$.

Fig. 13 displays the PV panel voltage and current sensed by Hall effect transducers. In case of the proposed $\mathrm{AbM}$, the result shows less oscillations and stable waveforms than the P\&O. It can be noted that the presence of oscillations with low frequencies in the current and voltage are mostly due to noises measurement [see Figs. 13(c) and (d)]. However, the controlled system using the AbM method has a good noise and fluctuations rejection around the MPP.
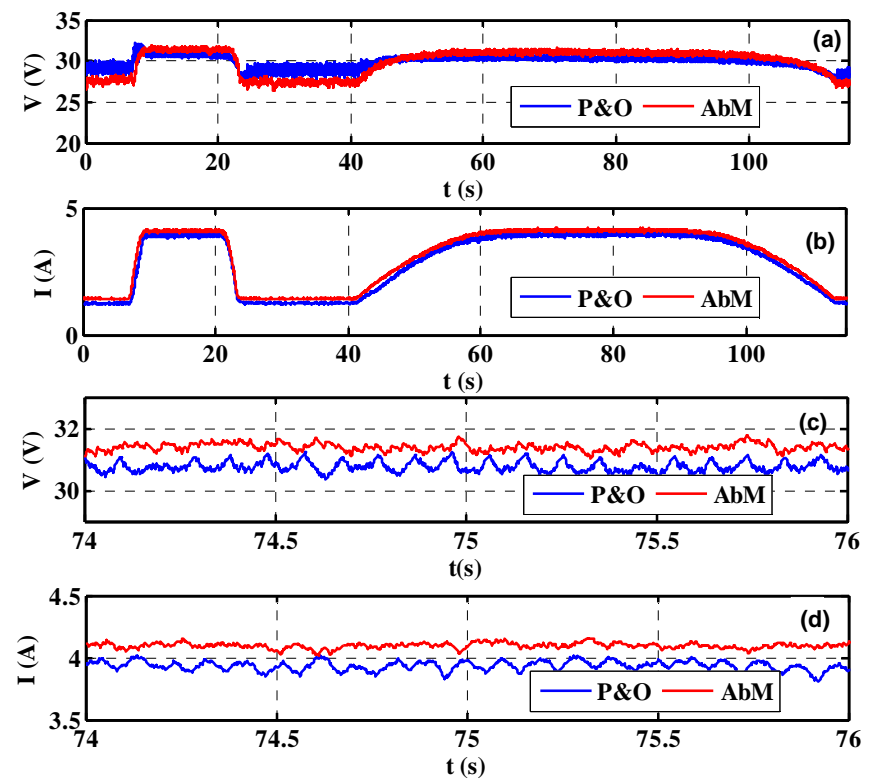

Fig. 13. Experimental comparison results of the $A b M$ and P\&O methods: (a) PV voltage; (b) PV current; (c) zoom on the PV voltage in steady state; (d) zoom on the PV current in steady state.

\section{CONCLUSION}

New MPPT concept has been proposed in this paper for improving maximum power extraction in PV systems. The proposal exploits the simple ADALINE structure which leads to easy and low-cost implementation. To verify its effectiveness, simulation and experiment have been conducted under variable solar irradiance and load. A comparison with the $\mathrm{P} \& \mathrm{O}$ algorithm has been conducted under the same conditions. The obtained results confirmed performances superiority of the proposed AbM algorithm which can be summarized as fallow:

1) $100 \%$ of power efficiency in steady and transient-states,

2) Zero oscillations in the PV current and voltage in steady-state,

3) Minor oscillations in the PV current and voltage in transient-state,

4) More easily implementable than the simpler $\mathrm{P} \& \mathrm{O}$ algorithm,

5) Analogic implementation is possible with basic circuits.

The suggested method is equally effective for low insolation levels and can works even where sudden solar irradiance changes are likely to occur. So, the proposal may be suitable for tracking uncommonly fast irradiance variations like mobile solar applications. Finally, due to reduced and absence of complex computations in the AbM scheme, it is an excellent candidate for increasing the reliability for commercial PV systems.

As future work, we intend to test the developed AbM using a real laboratory prototype based on several PV panels integrated into a more complex system such as a distributed generation system connected to a microgrid. The test setup can be used to evaluate the AbM performances regarding to multiple connected linear and non-linear loads. Other future scope is to test the suggested MPPT algorithm for drawing the maximum power from the PV modules in further applications such as single-stage grid-connected PV systems. In this application, experimental comparisons between the 
proposed AbM and other existing MPPTs will be performed especially under partial shading conditions.

\section{ACKNOWLEDGEMENT}

This work was supported by the Franco-Algerian cooperation program PHC-TASSILI (Project No. 17MDU995).

\section{REFERENCES}

[1] S. Messalti, A. Harrag, and A. Loukriz, "A new variable step size neural networks MPPT controller: Review, simulation and hardware implementation," Renewable and Sustainable Energy Reviews, vol. 82, Feb., pp. 1313-1319, 2018.

[2] A. Ba, C. O. Ehssein, M. E. M. O. M. Mahmoud, O. Hamdoun, and A. Elhassen, "Comparative study of different DC/DC power converter for optimal PV system using MPPT (P\&O) method," Applied Solar Energy, vol. 54, no. 4, Jul., pp. 235-245, 2018.

[3] C. Bharatiraja, S. Jeevananthan, and R. Latha, "FPGA based practical implementation of NPC-MLI with SVPWM for an autonomous operation PV system with capacitor balancing," International Journal of Electrical Power \& Energy Systems, vol. 61, Oct., pp. 489-509, 2014.

[4] R. J. Mukti, and A. Islam, "Modeling and performance analysis of PV module with maximum power point tracking in Matlab/Simulink," Applied Solar Energy, vol. 51, no. 4, Oct., pp. 245$252,2015$.

[5] D. C. Huynh, and M. W. Dunnigan, "Development and comparison of an improved incremental conductance algorithm for tracking the MPP of a solar PV panel," IEEE Transactions on Sustainable Energy, vol. 7, no. 4, Oct., pp. 1421-1429, 2016.

[6] M. M. Shebani, T. Iqbal, and J. E. Quaicoe, "Comparing bisection numerical algorithm with fractional short circuit current and open circuit voltage methods for MPPT photovoltaic systems," In Proc. IEEE Electrical Power and Energy Conference, 10, 2016, pp. 1-5.

[7] V.R. Kota, and M. N. Bhukya, "A novel linear tangents-based P\&O scheme for MPPT of a PV system," Renewable and Sustainable Energy Reviews, vol. 71, May, pp. 257-267, 2017.

[8] H. P. Desai, R. Maheshwari, S. N. Sharma, and V. Shah, "Maximum power extraction from photo-voltaic power generator with adaptive MPP tracker," Applied Solar Energy, vol. 46, no. 4, Dec., pp. 251-257, 2010.

[9] M. Lasheen, A. K. A. Rahman, M. Abdel-Salam, and S. Ookawara, "Adaptive reference voltage-based MPPT technique for PV applications", IET Renewable Power Generation, vol. 11, no. 5, May, pp. 715-722, 2017.

[10] J. Ahmed, and Z. Salam, "An enhanced adaptive P\&O MPPT for fast and efficient tracking under varying environmental conditions," IEEE Transactions on Sustainable Energy, vol. 9, no. 3, Jul., pp. 1487-1496, 2018.

[11] N. Kumar, I. Hussain, B. Singh, and B. K. Panigrahi, "Framework of maximum power extraction from solar PV panel using self-predictive perturb and observe algorithm," IEEE Transactions on Sustainable Energy, vol. 9, no. 2, Apr., pp. 895-903, 2018.

[12] A. Thangavelu, S. Vairakannu, and D. Parvathyshankar, "Linear open circuit voltage-variable step-size-incremental conductance strategy-based hybrid MPPT controller for remote power applications," IET Power Electronics, vol. 10, no. 11, Sep., pp. 1363-1376, 2017.

[13] S. Motahhir, A. Chalh, A. El Ghzizal, and A. Derouich, "Development of a Low-cost PV system using an improved INC algorithm and a PV panel Proteus model," Journal of Cleaner Production, vol. 204, Dec., pp. 355-365, 2018.

[14] A. Youssef, M. E. Telbany, and A. Zekry, "Reconfigurable generic FPGA implementation of fuzzy logic controller for MPPT of PV systems," Renewable and Sustainable Energy Reviews, vol. 82, no. 1, Feb., pp. 1313-1319, 2018.

[15] A. M. Farayola, A. N. Hasan, and A. Ali, "Implementation of modified incremental conductance and fuzzy logic MPPT techniques using MCUK converter under various environmental conditions," Applied Solar Energy, vol. 53, no. 2, Apr., pp. 173-184, 2017.

[16] Y. Kohata, K. Yamauchi, and M. Kurihara. "Quick maximum power point tracking of photovoltaic using online learning neural network", In Proc. International Conference Neural 
Information Processing, 2009, pp. 606-613.

[17] K. H. Chao, M. H. Wang, and Y. S. Lee, "An extension neural network based incremental MPPT method for a PV system," In Proc. International Conference on Machine Learning and Cybernetics, 2011, pp. 3021-3025.

[18] W. M. Lin, C. M. Hong, and C. H. Chen, "Neural-network-based MPPT control of a standalone hybrid power generation system," IEEE Transactions on Power Electronics, vol. 26, no. 12, Dec., pp. 3571-3581, 2011.

[19] Syafaruddin, E. Karatepe, and T. Hiyama, "Artificial neural network-polar coordinated fuzzy controller based maximum power-point tracking control under partially shaded conditions," IET Renewable Power Generation, vol. 3, no. 2, Jun., pp. 239-253, 2009.

[20] A. A. Kulaksız, and R. Akkaya, "A genetic algorithm optimized ANN-based MPPT algorithm for a stand-alone PV system with induction motor drive," Solar Energy, vol. 86, Sept., pp. 2366-2375, 2012.

[21] R. Subha, and S. Himavathi, "Active power control of a photovoltaic system without energy storage using neural network-based estimator and modified P\&O algorithm," IET Generation, Transmission \& Distribution, vol. 12, no. 4, Feb., pp. 927-934, 2018.

[22] A. I. Dounis, P. Kofinas, G. Papadakis, and C. Alafofimos, "A direct adaptive neural control for maximum power point tracking of photovoltaic system," Solar Energy, vol. 115, May, pp. 145-165, 2015.

[23] L. M. Elobaid, A. K. Abdelsalam, and E. E. Zakzouk, "Artificial neural network-based photovoltaic maximum power point tracking techniques: a survey," IET Renewable Power Generation, vol. 9, no. 8, Nov., pp.1043-1063, 2015.

[24] F. Paz, and M. Ordonez, "High-Performance solar MPPT using switching ripple identification based on a lock-in amplifier," IEEE Transactions on Industrial Electronics, vol. 63, no. 6, Jun., pp. 3595-3604, 2016.

[25] J. Ahmed, and Z. Salam, "A modified P\&O maximum power point tracking method with reduced steady-state oscillation and improved tracking efficiency," IEEE Transactions on Sustainable Energy, vol. 7, no. 4, Oct. pp. 1506-1515, 2016.

[26] X. Li, H. Wen, L. Jiang, W. Xiao, Y. Du, and C. Zhao, "An improved MPPT method for pv system with fast-converging speed and zero oscillation," IEEE Transactions on Industry Applications, vol. 52, no. 6., Nov., pp. 5051-5064, 2016.

[27] F. Paz, and M. Ordonez, "Zero oscillation and irradiance slope tracking for photovoltaic MPPT," IEEE Transactions on Industrial Electronics, vol. 61, no. 11, Nov., pp. 6138-6147, 2014.

[28] B. Widrow and M. A. Lehr, "30 years of adaptive neural networks: perceptron, MADALINE, and backpropagation," In Proc. IEEE, vol 78, no. 9, 1990, pp. 1415-1442.

[29] M. Rosu-Hamzescu, and S. Oprea, "Practical guide to implementing solar panel MPPT algorithms," Microchip Technology Inc, Application Note, AN152, 2013.

[30] M. A. Elgendy, B. Zahawi, and D. J. Atkinson, "Assessment of Perturb and Observe MPPT Algorithm Implementation Techniques for PV Pumping Applications," IEEE Transactions on Sustainable Energy, vol. 3, no. 1, Jan., pp. 21-33, 2012.

[31] A. Rahoui, A. Bechouche, H. Seddiki, and D. Ould Abdeslam, "Grid voltages estimation for three-phase PWM rectifiers control without AC voltage sensors," IEEE Transactions on Power Electronics, vol. 33, no. 1, Jan., pp. 859-875, 2018.

[32] Y. Triki, A. Bechouche, H. Seddiki, D. Ould Abdeslam and P. Wira, "ADALINE based maximum power point tracking methods for stand-alone PV system control," In Proc. IEEE International Conference on Industrial Technology, 02, 2018, pp. 880-885.

[33] M. Andresen, G. Buticchi, and M. Liserre, "Thermal stress analysis and MPPT optimization of photovoltaic systems," IEEE Transactions on Industrial Electronics, vol. 63, no. 8, Aug., pp. 4889-4898, 2016. 